\title{
Upgrade of the CMS muon spectrometer in the forward region with the GEM technology.
}

\author{
Marek Gruchała*a on behalf of the CMS Muon group. \\ ${ }^{a}$ Ghent University \\ Ghent, Belgium \\ E-mail: marek.gruchala@cern.ch
}

The Large Hadron Collider (LHC) will be upgraded in several phases that will allow the significant expansion of its physics program and the sustainability for the requirements to maintain sensitivity for the electroweak and TeV scales. After the expected long shutdown in 2018 (LS2) the accelerator luminosity will be increased to $2-3 \times 10^{34} \mathrm{~cm}^{-2} \mathrm{~s}^{-1}$ exceeding the design value of $1 \times 10^{34} \mathrm{~cm}^{-2} \mathrm{~s}^{-1}$ allowing the collection of the CMS experiment approximately $100 \mathrm{fb}^{-1} /$ year. A subsequent upgrade in 2022-23 will increase the luminosity up to $5 \times 10^{34} \mathrm{~cm}^{-2} \mathrm{~s}^{-1}$. To cope with the corresponding increase in background rates and trigger efficiency requirements, the installation of additional muon detectors is a necessity. Three major upgrades are planned for the CMS muon endcap regions, referred as GE1/1, GE2/1 and ME0. Each of the additional set of detectors is based on the Gas Electron Multiplier (GEM) technology, a choice based on many past R\&D activities. While the installation of the GE1/1 chambers has been already approved and scheduled by 2019/20, the GE2/1 project is in an advanced phase of design and the ME0 project is now in the final phase of review. We present an overview of the muon spectrometer upgrade based on GEM technology, the details of the ongoing GE1/1 chamber production with the first results of the quality assurance tests. Moreover, preliminary results obtained for the GE2/1 single module M4 will be shown along with the design and the technical solution adopted for the foreseen GE2/1 and ME0 chambers.

The 39th International Conference on High Energy Physics (ICHEP2018)

4-11 July, 2018

Seoul, Korea

${ }^{*}$ Speaker. 


\section{CMS GEM Project}

The GEM project is composed of three step high- $\eta$ region upgrade, referred here as GE1/1, GE2/1 and ME0. The baseline is installation of 36 double-layered triple-GEM Chambers, called GE1/1, in front of the ME1/1 station during the LS2. The new detection ring will provide full $\phi$ coverage and depending on the chamber size $1.55<|\eta|<2.18$ or $1.61<|\eta|<2.18$ in pseudorapidity for long and short version respectively.

The second upgrade, referred as GE2/1 will consist of 18 double-layered triple-GEM units, each composed of 8 different modules. The division into 8 different modules is necessary due to the foil production limitations and is done in the way that does not introduce dead spots to the unit as a whole. The GE2/1, similarly as GE1/1, will cover full azimuthal angle $\phi$ but different pseudorapidity range - 1.6 $<|\eta|<2.45$. The installation of the first endcap is scheduled for Q1 2022 while completion of the second endcap is scheduled for Q1 2023.

The third GEM-based project is a muon near-trigger (ME0) with 18 six-layered triple-GEM chamber stacks, each covering 20 degrees in $\phi$ and $2.0<|\eta|<3.5$ in pseudorapidity. In case of ME0 project the installation is scheduled for 2023/2024. The locations of each upgrades, mentioned in this section is shown on the figure 1 .

\section{GEM Technology}

Gas Electron Multiplier (GEM) is a Micro Pattern Gaseous Detector (MPGD) family member. It is composed of $50 \mu \mathrm{m}$ thick resistive polyimide foil, layered between two $5 \mu \mathrm{m}$ conductive layers, typically copper. The foil perforation follows the hexagonal pattern, with a typical hole diameter of $70 \mu \mathrm{m}$ and $140 \mu \mathrm{m}$ pitch [1].

The GEM technology presents various of important features, that make them an excellent choice for upcoming and future upgrades, such as a good spatial [2] and time resolution, an efficiency reaching $98 \%$ level and a high rate capability $[3,4]$. Moreover they are proven to be radiation hard detectors. Preceding set of features make them an ideal

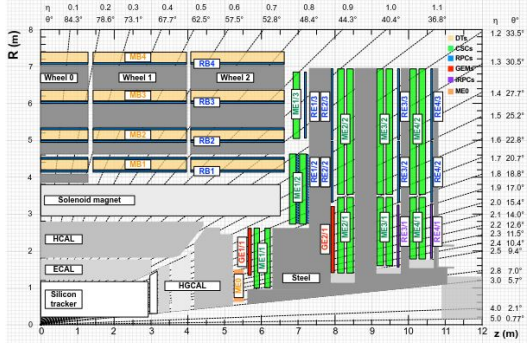

Figure 1: A quadrant of the $\mathrm{R}$ $\mathrm{z}$ cross-section of the CMS detector, highlighting in red the location of the proposed GE1/1 and GE2/1 detectors within the CMS muon system while the ME0 position is indicated with yellow color.

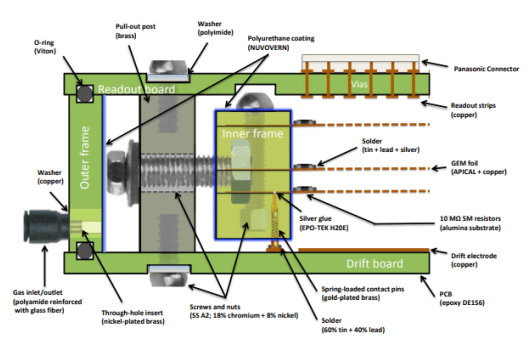

Figure 2: Cross section through inner and outer chamber frames and GEM foils. material for a triggering-tracking detector and consequently resulting in adoption of this technology in other experiments at CERN, such as LHCb, COMPASS and TOTEM.

\section{GE1/1 Design}


The GE1/1 detectors are composed of three GEM foils, sandwiched at their edges between four layers of a thin frame, made from a halogen-free glass epoxy. The thickness of the different frame layers determines the spacing between the foils as well as between the outer foils and drift/read out boards. Whole stack is bounded together with the help of numerous small stainless steel screws, penetrating all the frame and foils. In order to ensure a proper stretching of the GEM foils every few $\mathrm{cm}$ stainless steel nuts are embedded into the frames with the axes remaining perpendicular to the inner frame and GEM foil surfaces. These nuts counter stainless steel screws that are inserted into small brass posts, so-called "pull-outs", which are located within the gas volume. By manual tightening of the pull-out screws, the GEM foils in the stack are tensioned due to inner frame being pulled outwards (towards the pullouts). The gas tightness of the detector is ensured by using a large outer glass-epoxy frame machined from a single piece and placed around the tensioned GEM stack and the brass pull-outs with two O-rings on top and bottom side, adjoining two of the PCBs (Figure 2). The first PCB, called drift, features a single drift cathode on its inner side and a solid ground plate on the outside for shielding purposes of the chamber. It also provides connections to the external HV power supply. The other PCB, readout board, has 24 highdensity header connectors with 130-pins on its outside to interface 128 the radial readout strips on the inside to the hybrids that plug into the readout board from the outside.
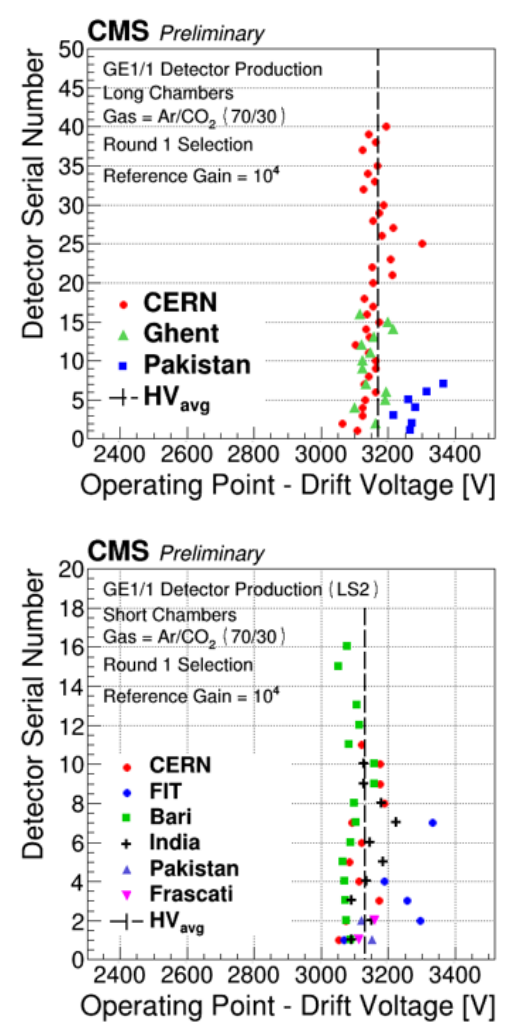

Figure 3: The determined HV operating point for every assembled long and short chambers respectively. The axis of ordinates represents the HV point at which each chamber form the axis of abscissa needs to operate in order to ensure equivalent gain of $10^{4}$.

\section{GE1/1 Production and Results}

The assembly and quality certification processes of the GE1/1 chambers are distributed among five sites. Each member of the site personnel involved in the assembly process has undergone a detailed training and is requested to follow strictly the assembly manual prepared by CERN in order to ensure the best quality and smooth operation of a chamber. After the assembly the chamber must undergo series of well defined quality checks(QC) [5] aiming to verify its properties of operation. The QC procedure is divided into two essential blocks, first related to single chamber physical operation, second aiming to verify super chamber (SC) ability for long term operation. The sites are requested to send the chambers that have successfully undergo the validation up to QC5 step included, to CERN. The post-assembly QC procedure consists of tests aiming to verify: chamber gas tightness (QC3), its long HV operation ability as well as intrinsic noise of each individual detector(QC4) and gain value as a function of applied voltage to the HV divider, that is used to 
power individual sections of the chamber, as well as the gain uniformity(QC5). The results of the QCs are summarized in figures 3(a),3(b),4(a) and 4(b).

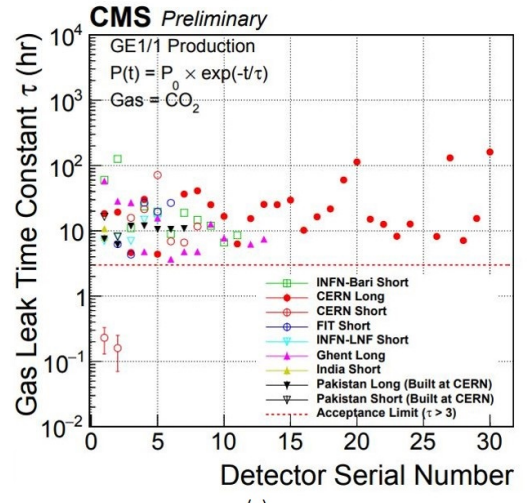

(a)

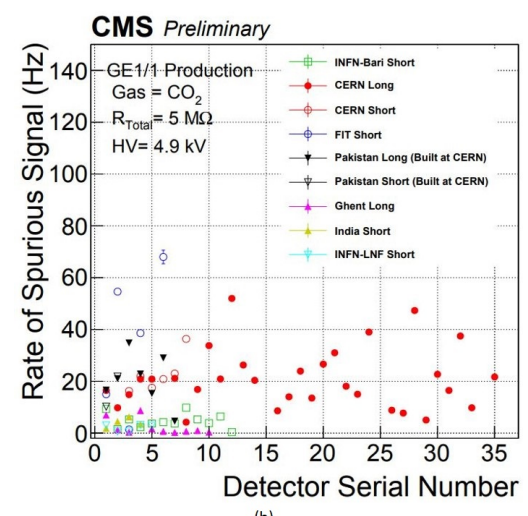

Figure 4: a)Gas leak parameters of the GE1/1 chambers. The leak parameter $\tau$ is defined by fitting the pressure vs time curve with $\operatorname{Pm}(\mathrm{t})=\operatorname{EXP}(\mathrm{A}-\mathrm{t} / \tau)$, where $\mathrm{A}$ is a constant. b)The rate of the intrinsic noise of the detector measured for the detectors assembled in specified sites in $\mathrm{Hz}$ vs. the serial number of the chamber. Here the intrinsic noise rate is being measured over whole surface of the GEM3 bottom electrode.

\section{Conclusions}

The production of the GE1/1 chambers has been distributed among 5 CERN certified production sites and CERN. Each of the sites had to prove its standalone ability to assemble and validate the chamber in a strict way that was supervised by CERN. Only after successful completion of all tests, the GE1/1 chamber kits for the upgrade detectors were sent to the institutes. As of the time this proceeding is being written all of the chambers that are needed to equip both, positive and negative endcaps have been assembled and most of them have been fully validated and are being prepared for a superchamber assembly.

\section{References}

[1] CMS Collaboration,"CMS Technical Design Report for Upgrade", CERN-LHCC-2015-012, CMS-TDR-013, CERN 2015, ISBN 978-92-9083-396-3.

[2] R.Wang et al., "A practical method to determine the spatial resolution of GEM detector", Nuclear Instruments and Methods in Physics Research Section A: Accelerators, Spectrometers, Detectors and Associated Equipment, Volume 701, 11 February 2013.

[3] D. Abbaneo et al., "Performance of a Large-Area GEM Detector Prototype for the Upgrade of the CMS Muon Endcap System", IEEE Nucl. Sci. Symp. Med. Imag. Conf. Rec.(2014) arXiv:1412.0228.

[4] M. Rossegnotti et al., "A new design using GEM-based technology for the CMS experiment", International Conference on Instrumentation for Colliding Beam Physics, Budker Institute of Nuclear Physics, Novosibirsk, Russia, 27 February - 3 March 2017, 2017 JINST 12 C07003.

[5] D. Abbaneo et al., "Quality control for the first large areas of triple-GEM chambers for the CMS endcaps", EPJ Web Conf., Volume 174, 2018, 4th International Conference on Micro Pattern Gaseous Detectors (MPGD 2015). 